\title{
Nanoparticle Chemical Composition
}

National Cancer Institute

\section{Source}

National Cancer Institute. Nanoparticle Chemical Composition. NCI Thesaurus. Code C62341.

The chemical elements of which a nanoparticle is composed. 